\title{
A new unbiased minimum variance observer for stochastic LTV systems with unknown inputs
}

\author{
LUC MEYeR* \\ ONERA, Université Paris Saclay, Chemin de la Huniere, 91120 Palaiseau, France \\ *Corresponding author. Email: luc.meyer@onera.fr \\ AND \\ Dalil IChalal and Vincent Vigneron \\ IBISC Lab, Université d'Evry-Val-d'Essonne, Université Paris Saclay, 43 Rue du Pelvoux, 91080 \\ Courcouronnes, France
}

[Received on 21 November 2017; revised on 29 December 2018; accepted on 14 February 2019]

\begin{abstract}
This paper is devoted to the state and input estimation of a linear time varying system in the presence of an unknown input (UI) in both state and measurement equations, and affected by Gaussian noises. The classical rank condition used in this kind of approach is relaxed in order to be able to be used in a wider range of systems. A state observer, that is an unbiased estimator with minimum error variance, is proposed. Then a UI observer is constructed, in order to be a best linear unbiased estimator, it follows a unique construction whether the direct feedthrough matrix is null or not. In a sense the proposed approach, generalizes and unifies the existing ones. Besides, a stability result is given for linear time invariant systems, which is a novelty for unbiased minimum variance observers relaxing the classical rank condition.
\end{abstract}

Keywords: state estimation; stochastic systems; unknown input; linear time varying systems; discretetime systems.

\section{Introduction}

Observation of the state of a dynamical system is one of the most important issues in control. For this reason, the developments of the Luenberg observer (Luenberger, 1971) (for deterministic systems), as well as the Kalman filter (Kalman, 1960) and its extensions for non-linear systems (Julier \& Uhlmann (1997); Jazwinski (2007)) (for stochastic systems), or the $H_{\infty}$ filtering were all a revolution in the control community. First applied on linear time invariant (LTI) systems, several variant of these observers have then been declined for linear time variant (LTV), or non-linear systems.

In the field of dynamical systems observers, systems with unknown inputs (UIs) are of a certain interest. These systems involve the presence of an UI on which there is no assumption: it can be an exogenous input as well as a non-linear function of the state for example.

In that field of research several works have been developed in deterministic case without any additional disturbance (Ichalal et al., 2009; Ichalal \& Mammar, 2015), via $L_{2}$ minimization when the disturbance is bounded (Pertew et al., 2005; Charandabi \& Marquez, 2014), or with an additional nonlinear Lipschtiz term in the state equation as in Yang et al. (2014), or via variance minimization when the system is perturbed by Gaussian white noise as in Darouach \& Zasadzinski (1997) and Darouach et al. (2003). 
This paper takes place in that last field of research. More precisely, it addresses the problem of estimating the state and the UI of a discrete-time LTV system in the presence of Gaussian noises, via the use of a minimum variance unbiased observer (as in Kalman Filtering, see Grewal, 2011). In this field of research, several works have been done. Darouach \& Zasadzinski (1997) has first proposed an efficient state observer for systems affected by UI in the state equation, and Darouach et al. (2003) has extended that work when the UI affects both state and measurement equations. Then Gillijns \& Moor (2007a) (resp. Gillijns \& Moor, 2007b) has proposed dual state and UI observers when the UI affects the state equation only (resp. both state and measurement equations). However, in that former work, the feedthrough matrix (the matrix associated with the UI in the measurement equation) has been assumed to be full column rank. Hsieh (2009a) has relaxed that last condition, but it has yield to a biased estimation of the UI. Cheng et al. (2009) gives necessary and sufficient conditions for the stability and the convergence of such a state observer. Wang et al. (2015) and Yong et al. (2016) both provide an unbiased minimum variance observer for state and UI estimation. In Ansari \& Bernstein (2018) and Maes et al. (2018), those results have been applied on maneuvering vehicle and structural dynamics. Song \& Zheng (2018b) and Song \& Zheng (2018a) have proposed a solution for state and input estimation when there are missing measurements. In Li et al. (2018), a new approach is proposed using an extended state in order to solve the estimation problem in a higher dimensional state before coming back to the initial state space. Finally, in Yong et al. (2014), the problem of state and UI estimation is considered using the entire time interval, and in Hsieh (2013), a filter for descriptor systems is provided.

The limit of the previous cited papers is that they are based on the assumption of a particular rank condition on system matrices. When that rank condition does not hold, those observers cannot be used anymore. Sundaram \& Hadjicostis (2006) has proposed a delayed observer that has relaxed that former rank condition. However, this method is not easy to implement, as it is based on some ad hoc constructions. Besides, it does not deliver any UI observer but only a state one. Finally, it is limited to the LTI case. Hsieh (2009b) has proposed an interesting alternative to the work of Sundaram \& Hadjicostis (2006). Based on the work done in Hsieh (2009a), it extends the rank condition, in order to be applied on the same classes of LTI systems with UI than Sundaram \& Hadjicostis (2006), but with a dual state and UI observer. Moreover, this latter work can also be applied on LTV systems. However, it does not propose a unique UI observer, but two different observers depending whether the feedthrough matrix is null or not. Besides none of the two previous works has made a stability analysis with convergence conditions, as with the proposed observer structures, such a analysis is not easy to deal with.

The aim of the present paper is to propose state and UI observers for all kinds of LTV systems even if the restrictive rank condition discussed above is not satisfied. In that sense, it overpasses most of the results discussed above, except the ones proposed in Sundaram \& Hadjicostis (2006) and Hsieh (2009b). In comparison to this two papers, this work gives an interesting alternative: it does not give any improvement in terms of performance, but the proposed observer structure enables to derive stability conditions (that has not been done before in Sundaram \& Hadjicostis, 2007 nor Hsieh, 2009b), and also to propose a unified alternative to Hsieh (2009b) for the UI estimation (Sundaram \& Hadjicostis, 2007 has not proposed any UI observer). Besides, the proposed observers take into account the presence of known input (which has not been the case in the two previous works).

This paper is divided as follows. After some preliminaries in Section 2, the mathematical frame of the observation problem is stated in Section 3. In Section 4, the state observer construction, as well as a stability analysis in the LTI case are given and proved. In Section 5, the same is done for the UI observer. In Section 6, some practical tools and remarks on the observer implementation are given (in particular, 
an algorithm is proposed). Finally, examples are given in Section 7 in order to illustrate the theoretical results.

\section{Preliminaries}

The set of real matrices with $n \times p$ elements is denoted by $\mathscr{M}_{n, p}(\mathbb{R})$. The subset of invertible matrices of $\mathscr{M}_{n, n}(\mathbb{R})$ is denoted by $G L_{n}(\mathbb{R}) . I_{n} \in \mathscr{M}_{n, n}(\mathbb{R})$ ( $I$ when there is no ambiguity) denotes the identity matrix and $0_{n, p} \in \mathscr{M}_{n, p}(\mathbb{R})(0$ when there is no ambiguity) denotes the null matrix with $n$ rows and $p$ columns. For any matrix $A \in \mathscr{M}_{n, p}(\mathbb{R}), A^{T}$ denotes its transpose, and $A^{\dagger}$ its pseudo-inverse. For any square matrix $A \in \mathscr{M}_{n, n}(\mathbb{R}), \operatorname{tr}(A)$ denotes its trace, and $\operatorname{det}(A)$ denotes its determinant. If $x$ is a stochastic vector, its expected value is denoted by $E[x]$.

For any vector sequence $v_{k}$ and any integer $r \geqslant 0$, let us set $v_{k: k+r}=\left[\begin{array}{llll}v_{k}^{T} & v_{k+1}^{T} & \ldots & v_{k+r}^{T}\end{array}\right]^{T}$. Finally, for $n \geqslant 1$ and $r \geqslant 1$, let us set $U_{n, r}=\left[\begin{array}{ll}I_{n} & 0_{n, r n}\end{array}\right]$.

Definition 2.1 Let be $x_{k}$ a stochastic signal. $\hat{x}_{k}$ is said to be an unbiased observer of $x_{k}$ if

$$
E\left[x_{k}-\hat{x}_{k}\right]=0, \quad \forall k \geqslant 0 .
$$

Note that, in particular, the state estimation error of an unbiased observer has to be independent from the UI when no assumption is made on that former.

Let recall that the state estimation error covariance matrix $P_{k}$ of a stochastic observer is given by $P_{k}=E\left[\left(x_{k}-\hat{x}_{k}\right)\left(x_{k}-\hat{x}_{k}\right)^{T}\right]$ for any $k \geqslant 0$. The variance of such an observer is given by the trace of this matrix.

\section{Problem Statement}

Let consider the following LTV system

$$
\left\{\begin{array}{l}
x_{k+1}=A_{k} x_{k}+B_{k} u_{k}+D_{k} d_{k}+F_{k} w_{k} \\
y_{k}=C_{k} x_{k}+E_{k} d_{k}+v_{k}
\end{array}\right.
$$

where $x_{k} \in \mathbb{R}^{n_{x}}, y_{k} \in \mathbb{R}^{n_{y}}, u_{k} \in \mathbb{R}^{n_{u}}$ and $d_{k} \in \mathbb{R}^{n_{d}}$ are the state vector, the output vector, the known input vector and the UI vector of the system, respectively. $\left(w_{k}\right)_{k \in \mathbb{N}} \subset \mathbb{R}^{n_{w}}$ and $\left(v_{k \subset \mathbb{N}}\right) \in \mathbb{R}^{n_{y}}$ are two sequences of independent zero-mean Gaussian noises whose covariance matrices sequences are denoted by $\left(W_{k}\right)_{k \in \mathbb{N}}$ and $\left(V_{k}\right)_{k \in \mathbb{N}}$, respectively. Moreover, the noises are assumed to be independent from $x_{0}$, whose covariance matrix is denoted by $P_{0}$. Finally, $A_{k}, B_{k}, C_{k}, D_{k}, E_{k}$ an $F_{k}$ are six time-varying matrices with appropriate dimensions.

Let be integers $r \geqslant 1$ and $k \geqslant 0$. A straightforward calculation gives the following equation (used for the main theorems proof):

$$
y_{k: k+r}=\mathscr{A}_{k: k+r} x_{k}+\mathscr{B}_{k: k+r} u_{r, k}+\mathscr{D}_{k: k+r} d_{r, k}+\mathscr{F}_{k: k+r} w_{k: k}+v_{k: k+r},
$$

where matrices $\mathscr{A}_{k: k+r}, \mathscr{B}_{k: k+r}, \mathscr{D}_{k: k+r}$ and $\mathscr{F}_{k: k+r}$ are given in (3.3). 
For the rest of the paper, the following notations will be used for any integer $r \geqslant 1$ and any $k \geqslant 0$ :

$$
\begin{aligned}
& \mathscr{W}_{k: k+r}=\left[\begin{array}{ccc}
W_{k} & & 0 \\
& \ldots & \\
0 & & W_{k+r}
\end{array}\right] \text { and } \mathscr{V}_{k: k+r}=\left[\begin{array}{ccc}
V_{k} & & 0 \\
& \cdots & \\
0 & & V_{k+r}
\end{array}\right] \text {. } \\
& \mathscr{A}_{k: k+r}=\left[\begin{array}{c}
C_{k} \\
C_{k+1} A_{k} \\
C_{k+2} A_{k+1} A_{k} \\
\ldots \\
C_{k+r} A_{k} \ldots A_{k+1} A_{k}
\end{array}\right] \\
& \mathscr{B}_{k: k+r}=\left[\begin{array}{cccccc}
0_{n_{y}, n_{u}} & 0_{n_{y}, n_{u}} & 0_{n_{y}, n_{u}} & \ldots & 0_{n_{y}, n_{u}} & 0_{n_{y}, n_{u}} \\
C_{k+1} B_{k} & 0_{n_{y}, n_{u}} & 0_{n_{y}, n_{u}} & \ldots & 0_{n_{y}, n_{u}} & 0_{n_{y}, n_{u}} \\
C_{k+2} A_{k+1} B_{k} & C_{k+2} B_{k+1} & 0_{n_{y}, n_{u}} & \ldots & 0_{n_{y}, n_{u}} & 0_{n_{y}, n_{u}} \\
\ldots & \ldots & \ldots & \ldots & \ldots & \ldots \\
C_{k+r} A_{k+r-1} \ldots A_{k+1} B_{k} & C_{k+r} A_{k+r-1} \ldots A_{k+2} B_{k+1} & \ldots & \ldots & C_{k+r} B_{k+r-1} & 0_{n_{y}, n_{u}}
\end{array}\right], \\
& \mathscr{D}_{k: k+r}=\left[\begin{array}{cccccc}
E_{k} & 0_{n_{y}, n_{d}} & 0_{n_{y}, n_{d}} & \ldots & 0_{n_{y}, n_{d}} & 0_{n_{y}, n_{d}} \\
C_{k+1} D_{k} & E_{k+1} & 0_{n_{y}, n_{d}} & \ldots & 0_{n_{y}, n_{d}} & 0_{n_{y}, n_{d}} \\
C_{k+2} A_{k+1} D_{k} & C_{k+2} D_{k+1} & E_{k+2} & \ldots & 0_{n_{y}, n_{d}} & 0_{n_{y}, n_{d}} \\
\ldots & \ldots & \ldots & \ldots & \ldots & \ldots \\
C_{k+r} A_{k+r-1} \ldots A_{k+1} D_{k} & C_{k+r} A_{k+r-1} \ldots A_{k+2} D_{k+1} & \ldots & \ldots & C_{k+r} D_{k+r-1} & E_{k+r}
\end{array}\right], \\
& \mathscr{F}_{k: k+r}=\left[\begin{array}{cccccc}
0_{n_{y}, n_{w}} & 0_{n_{y}, n_{w}} & 0_{n_{y}, n_{w}} & \ldots & 0_{n_{y}, n_{w}} & 0_{n_{y}, n_{w}} \\
C_{k+1} F_{k} & 0_{n_{y}, n_{w}} & 0_{n_{y}, n_{w}} & \ldots & 0_{n_{y}, n_{w}} & 0_{n_{y}, n_{w}} \\
C_{k+2} A_{k+1} F_{k} & C_{k+2} F_{k+1} & 0_{n_{y}, n_{w}} & \ldots & 0_{n_{y}, n_{w}} & 0_{n_{y}, n_{w}} \\
\ldots & \ldots & \ldots & \ldots & \ldots & \ldots \\
C_{k+r} A_{k+r-1} \ldots A_{k+1} F_{k} & C_{k+r} A_{k+r-1} \ldots A_{k+2} F_{k+1} & \ldots & \ldots & C_{k+r} F_{k+r-1} & 0_{n_{y}, n_{w}}
\end{array}\right]
\end{aligned}
$$

\section{State observer}

The aim of this section is to construct an unbiased state observer with minimum variance. The general form of such a linear observer, is

$$
\hat{x}_{k+1}=R_{k} \hat{x}_{k}+S_{k} u_{k: k+r}+K_{k} y_{k: k+r}
$$

The following assumption is useful in order to decouple the unknown input from the state estimation error.

Assumption 4.1 There exists an integer $r \geqslant 1$ such that for any integer $k \geqslant 0$, the following rank condition holds:

$$
\operatorname{rank}\left(\mathscr{D}_{k: k+r}\right)=\operatorname{rank}\left(\mathscr{D}_{k+1: k+r}\right)+\operatorname{rank}\left(\left[\begin{array}{c}
D_{k} \\
E_{k}
\end{array}\right]\right) .
$$

The following lemma gives another characterization of the rank condition given in Assumption 4.1. 
Lemma 4.1 Let be an integer $r \geqslant 1$, then for any $k \geqslant 0$, the following equation

$$
\operatorname{rank}\left(\left[\begin{array}{c}
\mathscr{D}_{k: k+r} \\
D_{k} U_{n_{d}, r}
\end{array}\right]\right)=\operatorname{rank}\left(\mathscr{D}_{k: k+r}\right)
$$

is equivalent to

$$
\operatorname{rank}\left(\mathscr{D}_{k: k+r}\right)=\operatorname{rank}\left(\mathscr{D}_{k+1: k+r}\right)+\operatorname{rank}\left(\left[\begin{array}{c}
D_{k} \\
E_{k}
\end{array}\right]\right) .
$$

Proof. See also Sundaram \& Hadjicostis (2007) for an alternative proof. For any $r \geqslant 1$ and $k \geqslant 0$, we have

$$
\begin{gathered}
{\left[\begin{array}{c}
\mathscr{D}_{k: k+r} \\
D_{k} U_{n_{d}, r}
\end{array}\right]=\left[\begin{array}{cc}
E_{k} & 0_{n_{y}, r n_{d}} \\
\Sigma_{k, r} D_{k} & \mathscr{D}_{k+1: k+r} \\
D_{k} & 0_{n_{x}, r n_{d}}
\end{array}\right]} \\
=\left[\begin{array}{ccc}
0 & I & 0 \\
\Sigma_{k, r} & 0 & I \\
I & 0 & 0
\end{array}\right]\left[\begin{array}{cc}
D_{k} & 0_{n_{x}, r n_{d}} \\
E_{k} & 0_{n_{y}, r n_{d}} \\
0_{r n_{y}, n_{d}} & \mathscr{D}_{k+1: k+r}
\end{array}\right], \\
\text { where } \Sigma_{k, r}=\left[\begin{array}{c}
C_{k+1} \\
C_{k+2} A_{k+1} \\
\ldots \\
C_{k+r} A_{k+r-1} \ldots A_{k+1}
\end{array}\right] . \text { The matrix }\left[\begin{array}{ccc}
0 & I & 0 \\
\Sigma_{k, r} & 0 & I \\
I & 0 & 0
\end{array}\right] \text { being clearly non singular, the rank }
\end{gathered}
$$

condition given by the lemma is established.

REMARK 4.1 Note that for $r=1$, equation (4.4) gives

$$
\operatorname{rank}\left(\left[\begin{array}{cc}
E_{k} & 0 \\
C_{k+1} D_{k} & E_{k+1}
\end{array}\right]\right)=\operatorname{rank}\left(\left[\begin{array}{l}
D_{k} \\
E_{k}
\end{array}\right]\right)+\operatorname{rank}\left(E_{k+1}\right),
$$

which is the rank condition widely required for UI decoupling (see for example Darouach et al., 2003 or Yong et al., 2016 for an alternative formulation). One characteristic of the proposed observer is precisely that it relaxes this condition thanks to the use of Assumption 4.1 and equation (3.2) for $r \geqslant 2$.

\subsection{Unbiased state observer}

REMARK 4.2 In order to make the observer unbiased when Assumption 4.1 holds but not for $r=1$, the use of successive measurements is required. Due to that fact, it is not easy to deal with the covariance calculation of the successive state estimation error (see Sundaram \& Hadjicostis, 2005 for more details on that issue).

The problem can be tackled in several ways. In Sundaram \& Hadjicostis (2007), a part of the state is estimated directly from the measurement vector, whereas the remaining part is estimated after several successive change of variables through a classical method of variance minimization (as in the Kalman filter). In Hsieh (2009b), a recursive three-step filter mixing both state and UI estimation is considered.

Our method is based on a judicious change of variable including the noises in a new extended state vector. Once that change of variable has been made, the sequel of the proof is based on the same 
techniques as for the Kalman filter. Thus, contrary to the previous works, the present filter derivation can easily lead to stability results (based on equivalent results for the Kalman filter).

Note that moreover, and contrary to Hsieh (2009b), the state estimation is totally independent from the UI estimation (Sundaram \& Hadjicostis, 2007 does not provide any UI observer). This means that if only the state estimation is needed, the part of the algorithm dealing with the UI estimation can be skipped, which can accelerate the filter execution.

TheOREM 4.1 Let Assumption 4.1 hold. Let us assume that $E\left[\hat{x}_{0}\right]=E\left[x_{0}\right]$. Then, equation (4.1) defines an unbiased observer for the state $x_{k}$ of system (3.1) if and only if its gains are given by

$$
\left\{\begin{array}{l}
R_{k}=A_{k}-K_{k} \mathscr{A}_{k: k+r} \\
S_{k}=B_{k} U_{r, n_{u}}-K_{k} \mathscr{B}_{k: k+r}, \\
K_{k}=G_{k}+X_{k} M_{k} H_{k}
\end{array}\right.
$$

where $G_{k}=D_{k} U_{r, n_{d}} \mathscr{D}_{k: k+r}^{\dagger}, H_{k}=I_{(r+1) n_{y}}-\mathscr{D}_{k: k+r} \mathscr{D}_{k: k+r}^{\dagger}, M_{k}$ and $X_{k}$ are any matrices with appropriate and compatible dimensions (they will be discussed in the sequel).

Besides, the state estimation error $e_{k}=x_{k}-\hat{x}_{k}$ satisfies the following dynamics:

$$
e_{k+1}=\left(A_{k}-K_{k} \mathscr{A}_{k: k+r}\right) e_{k}+\left(F_{k} U_{r-1, n_{w}}-K_{k} \mathscr{F}_{k: k+r}\right) w_{k: k+r}-K_{k} v_{k: k+r}
$$

Proof. The calculation of the error gives

$$
\begin{aligned}
e_{k+1}= & \left(A_{k}-K_{k} \mathscr{A}_{k: k+r}\right) e_{k}+\left(A_{k}-K_{k} \mathscr{A}_{k}-R_{k}\right) \hat{x}_{k}+\left(D_{k} U_{r, n_{d}}-K_{k} \mathscr{D}_{k: k+r}\right) d_{k: k+r} \\
& +\left(B_{k} U_{r, n_{u}}-S_{k}-K_{k} \mathscr{B}_{k: k+r}\right) u_{k: k+r}+\left(F_{k} U_{r, n_{w}}-K_{k} \mathscr{F}_{k: k+r}\right) w_{k: k+r}-K_{k} v_{k: k+r} .
\end{aligned}
$$

Based on Definition 2.1, the proposed observer is an unbiased one if and only if

$$
\forall k \geqslant 0, \quad E\left[e_{k}\right]=0 .
$$

In the present case, recalling that all noises have zero means, the expected value of $e_{k}$ satisfies

$$
\begin{aligned}
E\left[e_{k+1}\right]= & \left(A_{k}-K_{k} \mathscr{A}_{k: k+r}\right) E\left[e_{k}\right]+\left(A_{k}-K_{k} \mathscr{A}_{k: k+r}-R_{k}\right) E\left[\hat{x}_{k}\right] \\
& +\left(D_{k} U_{r, n_{d}}-K_{k} \mathscr{D}_{k: k+r}\right) E\left[d_{k: k+r}\right]+\left(B_{k} U_{r, n_{u}}-S_{k}-K_{k} \mathscr{B}_{k: k+r}\right) E\left[u_{k: k+r}\right] .
\end{aligned}
$$

Thus, in order to have an unbiased observer, and provided that $E\left[e_{0}\right]=0$ (i.e. $E\left[\hat{x}_{0}\right]=E\left[x_{0}\right]$ ), it is necessary and sufficient to satisfy the following conditions:

$$
\left\{\begin{array}{l}
R_{k}=A_{k}-K_{k} \mathscr{A}_{k: k+r} \\
S_{k}=B_{k} U_{r, n_{u}}-K_{k} \mathscr{B}_{k: k+r} \\
K_{k} \mathscr{D}_{k: k+r}=D_{k} U_{r, n_{d}}
\end{array} .\right.
$$


Using Assumption 4.1 and Lemma 4.1, the last equality has a general solution for $K_{k}$ given by

$$
K_{k}=G_{k}+X_{k} M_{k} H_{k}
$$

where $G_{k}=D_{k} U_{r, n_{d}} \mathscr{D}_{k: k+r}^{\dagger}, H_{k}=I_{(r+1) n_{y}}-\mathscr{D}_{k: k+r} \mathscr{D}_{k: k+r}^{\dagger}$ and $M_{k}$ and $X_{k}$ are any matrices with appropriate dimensions.

Finally, under Conditions (4.12) (or equivalently under conditions (4.7)), the state estimation error takes the form

$$
e_{k+1}=\left(A_{k}-K_{k} \mathscr{A}_{k: k+r}\right) e_{k}+\left(F_{k} U_{r-1, n_{w}}-K_{k} \mathscr{F}_{k: k+r}\right) w_{k: k+r}-K_{k} v_{k: k+r}
$$

which concludes the proof.

\subsection{Minimum variance state observer}

The aim of this subsection is to find the gain $X_{k}$ so that the covariance matrix of the state estimation error is with minimal trace.

THEOREM 4.2 Let us set $\mathscr{R}_{k}=\bar{B}_{k} \tilde{P}_{k} \bar{B}_{k}^{T}+\bar{D}_{k} \Sigma_{k+r} \bar{D}_{k}^{T}$. Let us assume that $\mathscr{R}_{k}$ is non-zero. Then among all the observers of the form (4.1) with gain matrices given by Theorem 4.1, the matrix $X_{k}$ leading to the minimum variance observer is given by

$$
X_{k}=\bar{I}^{T}\left(\bar{A}_{k} \tilde{P}_{k} \bar{B}_{k}^{T}+\bar{C}_{k} \Sigma_{k+r} \bar{D}_{k}^{T}\right) M_{k}^{T}\left(M_{k} \mathscr{R}_{k} M_{k}^{T}\right)^{-1}
$$

where

- $\tilde{P}_{k}$ evolves according to

$$
\tilde{P}_{k+1}=\left(\bar{A}_{k}-\bar{I} X_{k} M_{k} \bar{B}_{k}\right) \tilde{P}_{k}\left(\bar{A}_{k}-\bar{I} X_{k} M_{k} \bar{B}_{k}\right)^{T}+\left(\bar{C}_{k}-\bar{I} X_{k} M_{k} \bar{D}_{k}\right) \Sigma_{k+r}\left(\bar{C}_{k}-\bar{I} X_{k} M_{k} \bar{D}_{k}\right)^{T},
$$

$$
\text { where } \Sigma_{k+r}=\left[\begin{array}{cc}
W_{k+r} & 0 \\
0 & V_{k+r}
\end{array}\right] \text { and } \tilde{P}_{0}=\left[\begin{array}{ccc}
P_{0} & 0 & 0 \\
0 & \mathscr{W}_{0: r-1} & 0 \\
0 & 0 & \mathscr{V}_{0: r-1}
\end{array}\right] \text {, }
$$

- $M_{k}$ is such that $M_{k} \mathscr{R}_{k} M_{k}^{T}$ is non-singular (see Remark 4.4 for a possible way of constructing $M_{k}$ ),

- $\bar{I}=\left[\begin{array}{c}I_{n_{x}} \\ 0_{r n_{\eta}, n_{x}}\end{array}\right]$, with $n_{\eta}=n_{w}+n_{y}$,

- $\bar{A}_{k}, \bar{B}_{k}, \bar{C}_{k}$ and $\bar{D}_{k}$ are given in (4.17), in which $I_{n}^{l 1: l 2}$ stands for the sub matrix of $I_{n}$ composed by the columns from $l 1$ to $l 2$. 


$$
\begin{aligned}
& \bar{A}_{k}=\left[\begin{array}{ccccc}
A_{k}-G_{k} \mathscr{A}_{k: k+r} & F_{k}-G_{k} \mathscr{F}_{k: k+r} I_{(r+1) n_{w}}^{1: n_{w}} & -G_{k} \mathscr{F}_{k: k+r} I_{(r+1) n_{w}}^{n_{w}+1: r n_{w}} & -G_{k} I_{(r+1) n_{y}}^{1: n_{y}} & -G_{k} I_{(r+1) n_{y}}^{n_{y}+1: r n_{y}} \\
0_{(r-1) n_{w}, n_{x}} & 0_{(r-1) n_{w}, n_{w}} & I_{(r-1) n_{w}} & 0_{(r-1) n_{w}, n_{y}} & 0_{(r-1) n_{w},(r-1) n_{y}} \\
0_{n_{w}, n_{x}} & 0_{n_{w}, n_{w}} & 0_{n_{w},(r-1) n_{w}} & 0_{n_{w}, n_{y}} & 0_{n_{w},(r-1) n_{y}} \\
0_{(r-1) n_{y}, n_{x}} & 0_{(r-1) n_{y}, n_{w}} & 0_{(r-1) n_{y},(r-1) n_{w}} & I_{(r-1) n_{y}} & 0_{(r-1) n_{y}, n_{y}} \\
0_{n_{y}, n_{x}} & 0_{n_{y}, n_{w}} & 0_{n_{y},(r-1) n_{w}} & 0_{n_{y}, n_{y}} & 0_{n_{y},(r-1) n_{y}}
\end{array}\right] \\
& \bar{B}_{k}=\left[\begin{array}{lllll}
H_{k} \mathscr{A}_{k: k+r} & H_{k} \mathscr{F}_{k: k+r} I_{(r+1) n_{w}}^{1: n_{w}} \quad H_{k} \mathscr{F}_{k: k+r} I_{(r+1) n_{w}}^{n_{w}+1: n_{w}} & H_{k} I_{(r+1) n_{y}}^{1: n_{y}} & H_{k} I_{(r+1) n_{y}}^{n_{y}+1: r n_{y}}
\end{array}\right] \\
& \bar{C}_{k}=\left[\begin{array}{cc}
-G_{k} \mathscr{F}_{k: k+r} I_{(r+1) n_{w}}^{r n_{w}+1:(r+1) n_{w}} & -G_{k} I_{(r+1) n_{y}}^{r n_{y}+1:(r+1) n_{y}} \\
0_{(r-1) n_{w}, n_{w}} & 0_{(r-1) n_{w}, n_{y}} \\
I_{n_{w}} & 0_{n_{w}, n_{y}} \\
0_{(r-1) n_{y}, n_{w}} & 0_{(r-1) n_{y}, n_{y}} \\
0_{n_{y}, n_{w}} & I_{n_{y}}
\end{array}\right] \\
& \bar{D}_{k}=\left[\begin{array}{ll}
H_{k} \mathscr{F}_{k: k+r} I_{(r+1) n_{w}}^{r n_{w}+1:(r+1) n_{w}} & H_{k} I_{(r+1) n_{y}}^{r n_{y}+1:(r+1) n_{y}}
\end{array}\right] \text {, } \\
& \bar{E}_{k}=\left[\begin{array}{lll}
\mathscr{A}_{k: k+r} & \mathscr{F}_{k: k+r} I_{(r+1) n_{w}}^{1: r n_{w}} & I_{(r+1) n_{y}}^{1: r n_{y}}
\end{array}\right] \\
& \bar{F}_{k}=\left[\begin{array}{ll}
\mathscr{F}_{k: k+r} I_{(r+1) n_{w}}^{r n_{w}+1:(r+1) n_{w}} & I_{(r+1) n_{y}}^{r n_{y}+1:(r+1) n_{y}}
\end{array}\right]
\end{aligned}
$$

REMARK 4.3 If at a particular time step $k, \mathscr{R}_{k}$ is null, the minimum of the state estimation error does not exist uniquely. However, the user can set $X_{k}=0$ and continue to apply the observer at the next time step.

REMARK 4.4 A way of determining the matrix $M_{k}$ is the following. $\mathscr{R}_{k}$ being symmetric, its singular value decomposition takes the following form: $\mathscr{R}_{k}=U_{k}\left[\begin{array}{cc}\Gamma_{k} & 0 \\ 0 & 0\end{array}\right] U_{k}^{T}$, where $U_{k}$ is an orthogonal matrix and $\Gamma_{k}$ is a non-singular diagonal matrix. Let us set $M_{k}=\left[\begin{array}{ll}I_{\gamma_{k}} & 0\end{array}\right] U_{k}^{T}$, where $\gamma_{k}=\operatorname{rank}\left(\mathscr{R}_{k}\right)=$ $\operatorname{rank}\left(\Gamma_{k}\right)$. Then $M_{k} \mathscr{R}_{k} M_{k}^{T}=\Gamma_{k}$ is non-singular.

Let establish the proof of Theorem 4.2.

Proof. Let us rewrite equation (4.8) under the form

$$
\tilde{e}_{k+1}=\left(\bar{A}_{k}-\bar{I} X_{k} M_{k} \bar{B}_{k}\right) \tilde{e}_{k}+\left(\bar{C}_{k}-\bar{I} X_{k} M_{k} \bar{D}_{k}\right) \eta_{k+r}
$$

where $\tilde{e}_{k}=\left[\begin{array}{c}e_{k} \\ w_{k: k+r-1} \\ v_{k: k+r-1}\end{array}\right], \eta_{k+r}=\left[\begin{array}{c}w_{k+r} \\ v_{k+r}\end{array}\right], \bar{I}=\left[\begin{array}{c}I_{n_{x}} \\ 0_{r n_{\eta}, n_{x}}\end{array}\right], n_{\eta}=n_{w}+n_{y}$ and $\bar{A}_{k}, \bar{B}_{k}, \bar{C}_{k}$ and $\bar{D}_{k}$ are given in (4.17).

Now, recalling that $\left(w_{k}\right)_{k \in \mathbb{N}}$ and $\left(v_{k}\right)_{k \in \mathbb{N}}$ are sequences of independent Gaussian noises, it comes that $\eta_{k+r}$ and $\tilde{e}_{k}$ are independent stochastic signals (which is the reason why the previous transformation has 
been made). Besides, $e_{k}$ having zero mean (according to Theorem 4.1) and $w_{k+r}$ and $v_{k+r}$ having zero means (by assumption), it comes that $\tilde{e}_{k}$ and $\eta_{k+r}$ have zero means, and thus

$$
\tilde{P}_{k+1}=\left(\bar{A}_{k}-\bar{I} X_{k} M_{k} \bar{B}_{k}\right) \tilde{P}_{k}\left(\bar{A}_{k}-\bar{I} X_{k} M_{k} \bar{B}_{k}\right)^{T}+\left(\bar{C}_{k}-\bar{I} X_{k} M_{k} \bar{D}_{k}\right) \Sigma_{k+r}\left(\bar{C}_{k}-\bar{I} X_{k} M_{k} \bar{D}_{k}\right)^{T},
$$

where $\tilde{P}_{k}=\left[\begin{array}{cc}P_{k} & \Psi_{k} \\ \Psi_{k}^{T} & \Sigma_{k: k+r-1}\end{array}\right], \Sigma_{k+r}=\left[\begin{array}{cc}W_{k+r} & 0 \\ 0 & V_{k+r}\end{array}\right], \Sigma_{k: k+r-1}=\left[\begin{array}{cc}\mathscr{W}_{k: k+r-1} & 0 \\ 0 & \mathscr{V}_{k: k+r-1}\end{array}\right], P_{k}=$

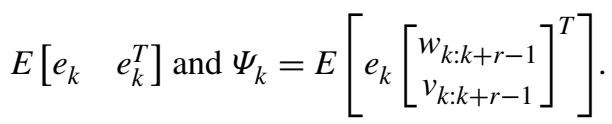

Now, the rest of the proof is devoted to the calculation of the gain $X_{k}$ that leads to the minimum variance of $e_{k}$, i.e. that minimizes $\operatorname{tr}\left(P_{k+1}\right)$. Then, $\operatorname{tr}\left(\tilde{P}_{k}\right)=\operatorname{tr}\left(P_{k}\right)+\operatorname{tr}\left(\Sigma_{k: k+r-1}\right)$, but $\Sigma_{k: k+r-1}$ being independent from the gain $X_{k}$, it leads that finding $X_{k}$ that minimizes $\operatorname{tr}\left(P_{k+1}\right)$ is equivalent to finding $X_{k}$ that minimizes $\operatorname{tr}\left(\tilde{P}_{k+1}\right)$.

In order to do that, let us calculate $\frac{\partial \operatorname{tr}\left(\tilde{P}_{k+1}\right)}{\partial X_{k}}$ and $\frac{\partial^{2} \operatorname{tr}\left(\tilde{P}_{k+1}\right)}{\partial X_{k}^{2}}$. Using derivation's matrices formulas from Petersen \& Pedersen (2008), it comes as

$$
\frac{\partial \operatorname{tr}\left(\tilde{P}_{k+1}\right)}{\partial X_{k}}=2 X_{k} M_{k} \mathscr{R}_{k} M_{k}^{T}-2 \bar{I}^{T}\left(\bar{A}_{k} \tilde{P}_{k} \bar{B}_{k}^{T}+\bar{C}_{k} \Sigma_{k+r} \bar{D}_{k}^{T}\right) M_{k}^{T}
$$

and

$$
\frac{\partial^{2} \operatorname{tr}\left(\tilde{P}_{k+1}\right)}{\partial X_{k}^{2}}=2 M_{k} \mathscr{R}_{k} M_{k}^{T}
$$

where $\mathscr{R}_{k}=\bar{B}_{k} \tilde{P}_{k} \bar{B}_{k}^{T}+\bar{D}_{k} \Sigma_{k+r} \bar{D}_{k}^{T}$. It is clear that $\frac{\partial^{2} \operatorname{tr}\left(\tilde{P}_{k+1}\right)}{\partial X_{k}^{2}}$ is a non-negative symmetric matrix. Then let us choose the matrix $M_{k}$ such that $M_{k} \mathscr{R}_{k} M_{k}^{T}$ is non-singular (a way of doing it is given in Remark 4.4). Then, it comes that $\frac{\partial^{2} \operatorname{tr}\left(\tilde{P}_{k+1}\right)}{\partial X_{k}^{2}}$ is a positive symmetric definite matrix, and thus, there exists a unique solution $X_{k}$ that minimizes $\operatorname{tr}\left(\tilde{P}_{k+1}\right)$, solution satisfying $\frac{\partial \operatorname{tr}\left(\tilde{P}_{k+1}\right)}{\partial X_{k}}=0$. Hence, it leads to the following value for $X_{k}$ :

$$
X_{k}=\bar{I}^{T}\left(\bar{A}_{k} \tilde{P}_{k} \bar{B}_{k}^{T}+\bar{C}_{k} \Sigma_{k+r} \bar{D}_{k}^{T}\right) M_{k}^{T}\left(M_{k} \mathscr{R}_{k} M_{k}^{T}\right)^{-1}
$$

REMARK 4.5 It is important to note that the variance is minimized from each time step to the next one: the variance minimization is local. Thus, there is no reason to obtain a global minimum variance observer. However, in some cases, that way of doing can lead to an optimal solution (as for the Kalman filter, see in Anderson \& Moore, 1979). But, in the present case, it is not the case: the local optimization leads to suboptimal global solution. This is due to the use of the extended state vector $\tilde{e}_{k}$ composed by the state estimation error but also by the noises. This point is explained in detail in Anderson \& Moore (1979), page 300.

Note that in Sundaram \& Hadjicostis (2007) and Hsieh (2009b), the proposed observer are also suboptimal, as they are also constructed from time step to time step with a local variance minimization 
goal. As a consequence, and as the local minimum in each time step in unique (see the proof of Theorem 4.2), the three observers give the same state estimation and Hsieh (2009b) as well as the observer proposed in this paper lead to the same UI estimation (see the example's results). However, the three algorithms proposed in the papers for the state and UI estimation are very different.

Then, again, the main contribution of this paper is not in terms of performance. The contribution is first to unify the estimating method for UI estimation (Sundaram \& Hadjicostis, 2007 has not proposed any UI estimation method, and Hsieh, 2009b has proposed two different algorithms depending on whether the matrix $E_{k}$ is null or not). The second contribution is the stability results given in the next subsection. This has not been done in previous works (probably due to the complex approaches of previous observers that cannot easily lead to the derivation of stability conditions).

\subsection{Stability property in LTI case}

Let consider the LTI system associated with system (3.1). The aim of the present subsection is to derive conditions for the convergence of matrix $\tilde{P}_{k}$.

In order to do so, let assume that matrix $\bar{D} \Sigma \bar{D}$ is non-null. This guarantee the possible construction of a constant matrix $M$ such that the inversion needed in the calculation of $\tilde{P}_{k+1}$ from $\tilde{P}_{k}$ is always possible (replace $\mathscr{R}_{k}$ by $\bar{D} \Sigma \bar{D}$ in the construction proposed in Remark 4.4).

- First of all, let recall the dynamics of $\tilde{P}_{k}$ given in equation (4.16):

$$
\tilde{P}_{k+1}=\left(\bar{A}-\bar{I} X_{k} M \bar{B}\right) \tilde{P}_{k}\left(\bar{A}-\bar{I} X_{k} M \bar{B}\right)^{T}+\left(\bar{C}-\bar{I} X_{k} M \bar{D}\right) \Sigma\left(\bar{C}-\bar{I} X_{k} M \bar{D}\right)^{T},
$$

- Let us set $\bar{X}_{k}=\bar{I} X_{k}$. Then equation (4.23) can be written as

$$
\tilde{P}_{k+1}=\left(\bar{A}-\bar{X}_{k} M \bar{B}\right) \tilde{P}_{k}\left(\bar{A}-\bar{X}_{k} M \bar{B}\right)^{T}+\left(\bar{C}-\bar{X}_{k} M \bar{D}\right) \Sigma\left(\bar{C}-\bar{X}_{k} M \bar{D}\right)^{T} .
$$

- By setting

$$
\left\{\begin{array}{l}
S=\bar{C} \Sigma \bar{D}^{T} M^{T} \\
\Theta=M \bar{D} \Sigma \bar{D}^{T} M^{T}, \\
Q=\bar{C} \Sigma \bar{C}^{T}
\end{array}\right.
$$

and then by setting $A_{s}=\bar{A}-S \Theta^{-1} M \bar{B}, B_{s}=M \bar{B}, Q_{s}=Q-S \Theta^{-1} S^{T}$ and $K_{k}=\bar{X}_{k}-S \Theta^{-1}$, it comes as

$$
\tilde{P}_{k+1}=\left(A_{s}-K_{k} B_{s}\right) \tilde{P}_{k}\left(A_{s}-K_{k} B_{s}\right)^{T}+K_{k} \Theta K_{k}^{T}+Q_{s} .
$$

- Finally, using the well-known results for the Kalman Filter (available for example in Anderson \& Moore, 1979), the following theorem holds.

THEOREM 4.3 With the notations introduced previously, and under the following conditions:

- the pair $\left(A_{s}, B_{s}\right)$ is detectable,

- the pair $\left(A_{s}, Q_{s}^{1 / 2}\right)$ is stabilizable, 
the covariance matrix $\tilde{P}_{k}$ given in Theorem 4.2 converges to the stabilizing solution $\tilde{P}$ of the following algebraic Riccati equation:

$$
\tilde{P}=\left(A_{s}-K B_{s}\right) \tilde{P}\left(A_{s}-K B_{s}\right)^{T}+K \Theta K^{T}+Q_{s} .
$$

As a consequence, the state estimation error $e_{k}=x_{k}-\hat{x}_{k}$ of the state observer introduced in Theorems 4.1 and 4.2 converges in mean-square to a constant positive bound.

\section{UI Observer}

In this section, the best linear unbiased estimator (BLUE) is constructed for UI estimation.

From equation (3.2), it comes that

$$
y_{k: k+r}=\mathscr{A}_{k: k+r} x_{k}+\mathscr{B}_{k: k+r} \tilde{u}_{k: k+r}+\mathscr{D}_{k: k+r} \tilde{d}_{k: k+r}+\mathscr{F}_{k: k+r} \tilde{w}_{k: k+r}+v_{k: k+r}
$$

By rewriting it, it comes:

$$
z_{k}=\mathscr{D}_{k: k+r} d_{k: k+r}+\epsilon_{k}
$$

where $z_{k}=y_{k: k+r}-\mathscr{B}_{k: k+r} u_{k: k+r}-\mathscr{A}_{k: k+r} \hat{x}_{k}$ is only made by known estimation, inputs and measurements and

$$
\epsilon_{k}=\mathscr{A}_{k: k+r} e_{k}+\mathscr{F}_{k: k+r} w_{k: k+r}+v_{k: k+r}
$$

Then $\epsilon_{k}$ can be rewritten under the following form:

$$
\epsilon_{k}=\bar{E}_{k} \tilde{e}_{k}+\bar{F}_{k} \eta_{k+r}
$$

where matrices $\bar{E}_{k}$ and $\bar{F}_{k}$ are given in (4.17). It is clear that $\epsilon_{k}$ is a Gaussian signal (as a linear combination of Gaussian signals) with expected value equal to $E\left[\epsilon_{k}\right]=0$ (as $E\left[e_{k}\right]=0, E\left[\tilde{w}_{k: k+r}\right]=0$ and $E\left[\tilde{v}_{k: k+r}\right]=0$ ), and covariance matrix equal to

$$
P_{\epsilon_{k}}=\bar{E}_{k} \tilde{P}_{k} \bar{E}_{k}^{T}+\bar{F}_{k} \Sigma_{k+r} \bar{F}_{k}^{T}
$$

In order to establish the UI observer, let us consider the following equation

$$
\hat{d}_{k}=L_{k} z_{k}
$$

Let us look for the gain $L_{k}$ leading to the BLUE. Both following theorems will be necessary in order to achieve the desired result. Before establishing them, let us make the following assumption.

Assumption 5.1. There exists an integer $r \geqslant 1$ such that for any $k \geqslant 0$ the following rank condition holds

$$
\operatorname{rank}\left(\mathscr{D}_{k: k+r}\right)=\operatorname{rank}\left(\mathscr{D}_{k+1: k+r}\right)+n_{d} .
$$

Assumption 5.1 is needed in order to estimate the UI (as it will be seen in the proof of Theorem 5.1). The following lemma gives another characterization of the rank condition given in Assumption 5.1. 
LEMMA 5.1 For any $r \geqslant 1$, the following equation

$$
\operatorname{rank}\left(\left[\begin{array}{c}
\mathscr{D}_{k: k+r} \\
U_{n_{d}, r}
\end{array}\right]\right)=\operatorname{rank}\left(\mathscr{D}_{k: k+r}\right)
$$

is equivalent to

$$
\operatorname{rank}\left(\mathscr{D}_{k: k+r}\right)=\operatorname{rank}\left(\mathscr{D}_{k+1: k+r}\right)+n_{d}
$$

Proof. For any $r \geqslant 1$ and any $k \geqslant 0$, we have

$$
\begin{aligned}
{\left[\begin{array}{c}
\mathscr{D}_{k: k+r} \\
U_{n_{d}, r}
\end{array}\right] } & =\left[\begin{array}{cc}
E_{k} & 0_{n_{y}, r n_{d}} \\
\Sigma_{k, r} D_{k} & \mathscr{D}_{k+1: k+r} \\
I_{n_{x}} & 0_{n_{x}, r n_{d}}
\end{array}\right] \\
& =\left[\begin{array}{ccc}
I_{n_{y}} & 0 & E_{k} \\
0 & I_{r n_{y}} & \Sigma_{k, r} D_{k} \\
0 & 0 & I_{n_{d}}
\end{array}\right]\left[\begin{array}{cc}
0_{n_{y}, n_{d}} & 0_{n_{y}, r n_{d}} \\
0_{r n_{y}, n_{d}} & \mathscr{D}_{k+1: k+r} \\
I_{n_{d}} & 0_{n_{d}, r n_{d}}
\end{array}\right],
\end{aligned}
$$

where $\Sigma_{k, r}=\left[\begin{array}{c}C_{k+1} D_{k} \\ C_{k+2} A_{k+2} D_{k} \\ \ldots \\ C_{k+r} A_{k+r-1} \ldots A_{k+1} D_{k}\end{array}\right]$. The matrix $\left[\begin{array}{ccc}I_{n_{y}} & 0 & E_{k} \\ 0 & I_{r n_{y}} & \Sigma_{k, r} D_{k} \\ 0 & 0 & I_{n_{d}}\end{array}\right]$ being clearly non singular, the rank condition given by the lemma is proved.

\subsection{Unbiased UI Observer}

Theorem 5.1 Let Assumption 5.1 hold. Then, equation (5.6) defines an unbiased observer for the UI $d_{k}$ of system (3.1) if and only if the gain $L_{k}$ takes the following form:

$$
L_{k}=J_{k}+Z_{k} N_{k} H_{k}
$$

where $J_{k}=U_{n_{d}, r} \mathscr{D}_{k: k+r}^{\dagger}, H_{k}=I_{(r+1) n_{y}}-\mathscr{D}_{k: k+r} \mathscr{D}_{k: k+r}^{\dagger}$, and matrices $Z_{k}$ and $N_{k}$ are any matrices with appropriate and compatible dimensions (they will be discussed in the sequel). Furthermore, the UI estimation error covariance matrix $P_{d_{k}}=E\left[e_{d_{k}} e_{d_{k}}^{T}\right]$ (where $e_{d_{k}}=d_{k}-\hat{d}_{k}$ is the UI estimation error) is given by

$$
P_{d_{k}}=L_{k} P_{\epsilon_{k}} L_{k}^{T}
$$

Proof. The UI estimation error $e_{d_{k}}=d_{k}-\hat{d}_{k}$ gives

$$
\begin{aligned}
e_{d_{k}} & =d_{k}-\hat{d}_{k} \\
& =U_{r, n_{d}} d_{k: k+r}-L_{k} z_{k} \\
& =U_{r, n_{d}} d_{k: k+r}-L_{k}\left(\mathscr{D}_{k: k+r} d_{k: k+r}+\epsilon_{k}\right) \\
& =\left(U_{r, n_{d}}-L_{k} \mathscr{D}_{k: k+r}\right) d_{k: k+r}-L_{k} \epsilon_{k}
\end{aligned}
$$

Based on Definition 2.1, the proposed UI observer (5.6) is an unbiased one if and only if

$$
\forall k \geqslant 0, \quad E\left[e_{d_{k}}\right]=0 .
$$


In the present case, recalling that $E[\epsilon]=0$, the expected value gives

$$
E\left[e_{d_{k}}\right]=\left(U_{r, n_{d}}-L_{k} \mathscr{D}_{k: k+r}\right) E\left[d_{k: k+r}\right] .
$$

Thus, in order to obtain an unbiased observer, it is necessary and sufficient to satisfy the following condition:

$$
L_{k} \mathscr{D}_{k: k+r}=U_{r, n_{d}},
$$

which, under Assumption 5.1 and thanks to Lemma 5.1, has a solution for $L_{k}$ given by (5.11). Finally, the UI estimation error becomes

$$
e_{d_{k}}=-L_{k} \epsilon_{k},
$$

which gives the covariance matrix given in the theorem's statement, and concludes the proof.

\subsection{Minimum Variance UI Observer}

Theorem 5.2 Let Assumption 5.7 hold. Let us set $\mathscr{S}_{k}=H_{k} P_{\epsilon_{k}} H_{k}^{T}$. Let us assume that $\mathscr{S}_{k} \neq 0$. Then, among all the observers under the form (5.6) with a gain $L_{k}$ given in (5.11), the one leading to the BLUE has the following gain $Z_{k}$ :

$$
Z_{k}=-J_{k} P_{\epsilon_{k}} H_{k}^{T} N_{k}^{T}\left(N_{k} \mathscr{S}_{k} N_{k}^{T}\right)^{-1}
$$

where matrix $N_{k}$ is chosen in order to make the matrix $N_{k} \mathscr{S}_{k} N_{k}^{T}$ non-singular (a possible way of constructing $N_{k}$ is given in Remark 5.3).

Remark 5.1 In Theorem 5.2, the term 'Best' in BLUE means that the proposed observer has the lowest variance as compared to the other unbiased observers.

Remark 5.2 If at a particular time step $k, \mathscr{S}_{k}$ is null, the minimum of the UI estimation error does not exist uniquely. However, the user can set $Z_{k}=0$ and continue to apply the observer at the next time step.

REMARK 5.3 A way of determining the matrix $N_{k}$ is the following. $\mathscr{S}_{k}$ being symmetric, its singular value decomposition takes the following form: $\mathscr{S}_{k}=U_{k}\left[\begin{array}{cc}\Gamma_{k} & 0 \\ 0 & 0\end{array}\right] U_{k}^{T}$, where $U_{k}$ is an orthogonal matrix and $\Gamma_{k}$ is a non-singular diagonal matrix. Let us set $N_{k}=\left[\begin{array}{ll}I_{\gamma_{k}} & 0\end{array}\right] U_{k}^{T}$, where $\gamma_{k}=\operatorname{rank}\left(\mathscr{S}_{k}\right)=\operatorname{rank}\left(\Gamma_{k}\right)$. Then $N_{k} \mathscr{S}_{k} N_{k}^{T}=\Gamma_{k}$ is non-singular.

Let establish the proof of Theorem 5.2.

Proof. Under the conditions of Theorem 5.1, the UI estimation covariance matrix is given by

$$
P_{d_{k}}=\left(J_{k}+Z_{k} N_{k} H_{k}\right) P_{\epsilon_{k}}\left(J_{k}+Z_{k} N_{k} H_{k}\right)^{T}
$$

Looking for the observer leading to the BLUE is equivalent to look for the gain $Z_{k}$ that gives the minimum variance, or equivalently to the gain $Z_{k}$ that gives the minimum value for the trace of the covariance matrix. It comes as

$$
\frac{\partial \operatorname{tr}\left(P_{d_{k}}\right)}{\partial Z_{k}}=2 Z_{k} N_{k} \mathscr{S}_{k} N_{k}^{T}+2 J_{k} P_{\epsilon_{k}} H_{k}^{T} N_{k}^{T},
$$


and

$$
\frac{\partial^{2} \operatorname{tr}\left(P_{d_{k}}\right)}{\partial Z_{k}^{2}}=2 N_{k} \mathscr{S}_{k} N_{k}^{T},
$$

where $\mathscr{S}_{k}=H_{k} P_{\epsilon_{k}} H_{k}^{T}$.

It is clear that $\frac{\partial^{2} \operatorname{tr}\left(P_{d_{k}}\right)}{\partial Z_{k}^{2}}$ is a non-negative symmetric matrix. Besides, $N_{k}$ is chosen in order to make it non-singular. Thus, $\frac{\partial^{2} \operatorname{tr}\left(P_{d_{k}}\right)}{\partial Z_{k}^{2}}$ is a positive definite matrix, and hence there exists a unique gain $Z_{k}$ that minimizes the trace of the covariance matrix $P_{d_{k}}$, and this gain is solution of the following equation:

$$
\frac{\partial \operatorname{tr}\left(P_{d_{k}}\right)}{\partial Z_{k}}=0 .
$$

Finally, it comes as

$$
Z_{k}=-J_{k} P_{\epsilon_{k}} H_{k}^{T} N_{k}^{T}\left(N_{k} \mathscr{S}_{k} N_{k}^{T}\right)^{-1}
$$

which concludes the proof.

REMARK 5.4 Let us consider the LTI case, and let us discuss the stability of the matrices sequence $\left(P_{d_{k}}\right)_{k \in \mathbb{N}}$. Let us assume that $H \bar{F} \Sigma \bar{F}^{T} H^{T}$ is non-null. Then it is possible to construct a matrix $N$ such that $N \mathscr{S}_{k} N^{T}$ is non-singular (recall that $\mathscr{S}_{k}=H_{k} P_{\epsilon_{k}} H_{k}^{T}$ ). Now, using previous results, it comes that $P_{d_{k}}$ can be expressed as

$$
\begin{aligned}
P_{d_{k}} & =L_{k} P_{\epsilon_{k}} L_{k}^{T} \\
& =\left(J+Z_{k} N H\right) P_{\epsilon_{k}}\left(J+Z_{k} N H\right)^{T} \\
& =\left(J-J P_{\epsilon_{k}} H^{T} N^{T}\left(N \mathscr{S} N^{T}\right)^{-1} N H\right) P_{\epsilon_{k}}\left(J-J P_{\epsilon_{k}} H^{T} N^{T}\left(N \mathscr{S} N^{T}\right)^{-1} N H\right)^{T} \\
& =\left(J-J P_{\epsilon_{k}} H^{T} N^{T}\left(N H P_{\epsilon_{k}} H^{T} N^{T}\right)^{-1} N H\right) P_{\epsilon_{k}}\left(J-J P_{\epsilon_{k}} H^{T} N^{T}\left(N H P_{\epsilon_{k}} H^{T} N^{T}\right)^{-1} N H\right)^{T} \\
& =J P_{\epsilon_{k}} J^{T}-J P_{\epsilon_{k}} H^{T} N^{T}\left(N H P_{\epsilon_{k}} H^{T} N^{T}\right)^{-1} N H P_{\epsilon_{k}} J^{T} .
\end{aligned}
$$

The previous considerations show that, if the sequence $\left(P_{\epsilon_{k}}\right)_{k \in \mathbb{N}}$ is stable, then it is also the case of the sequence $\left(P_{d_{k}}\right)_{k \in \mathbb{N}}$. Besides, equation (5.5) gives

$$
P_{\epsilon_{k}}=\bar{E} \tilde{P}_{k} \bar{E}^{T}+\bar{F} \Sigma \bar{F}^{T}
$$

Therefore, if the sequence $\left(\tilde{P}_{k}\right)_{k \in \mathbb{N}}$ is stable, it is also the case of the sequence $\left(P_{d_{k}}\right)_{k \in \mathbb{N}}$. Hence, under the condition of stability and convergence on matrix $\tilde{P}_{k}$ given by Theorem 4.3 , the sequence $\left(P_{d_{k}}\right)_{k \in \mathbb{N}}$ is also stable and converges to the matrix $P_{d}$ given by

$$
P_{d}=J P_{\epsilon} J^{T}-J P_{\epsilon} H^{T} N^{T}\left(N H P_{\epsilon} H^{T} N^{T}\right)^{-1} N H P_{\epsilon} J^{T},
$$

where $P_{\epsilon}=\bar{E} \tilde{P} \bar{E}^{T}+\bar{F} \Sigma \bar{F}^{T}$, and $\tilde{P}$ is the limit of convergence of $\tilde{P}_{k}$. 


\section{AlgORITHM 6.1. State and UI observer algorithm}

Determination of the decoupling degree $r \geqslant 1$.

Initialize the value of $\hat{x}_{0}$.

Initialize the value of $\left.\left.P_{0}=E\left[\left(x_{0}-\hat{x}_{0}\right)\right)^{T}\left(x_{0}-\hat{x}_{0}\right)\right)\right]$ (take a high value if not known).

for $\% k \geqslant 0$ do

$\%$ State observer

$G_{k}=D_{k} U_{n_{d}, r}\left(\mathscr{D}_{k: k+r}\right)^{+}$

$H_{k}=I_{(r+1) n_{y}}-\mathscr{D}_{k: k+r} \mathscr{D}_{k: k+r}^{+}$

Calculate $\bar{A}_{k}, \bar{B}_{k}, \bar{C}_{k}, \bar{D}_{k}, \bar{E}_{k}$ and $\bar{F}_{k}$ with notations (4.17)

$\Sigma_{k+r}=\left[\begin{array}{ll}W_{k+r} & 0 \\ 0 & W_{k+r}\end{array}\right]$

$\mathscr{R}_{k}=\bar{B}_{k} \tilde{P}_{k} \bar{B}_{k}^{T}+\bar{D}_{k} \Sigma_{k+r} \bar{D}_{k}^{T}$

Determine of $M_{k}$ according to remark 4.4

$X_{k}=\bar{I}^{T}\left(\bar{A}_{k} \tilde{P}_{k} \bar{B}_{k}^{T}+\bar{C}_{k} \Sigma_{k+r} \bar{D}_{k}^{T}\right) M_{k}^{T}\left(M_{k} \mathscr{R}_{k} M_{k}^{T}\right)^{-1}$

$K_{k}=G_{k}+X_{k} M_{k} H_{k}$

$S_{k}=B_{k} U_{r, n_{u}}-K_{k} \mathscr{B}_{k: k+r}$

$R_{k}=A_{k}-K_{k} \mathscr{A}_{k: k+r}$

$\hat{x}_{k}=R_{k} \hat{x}_{k}+S_{k} \tilde{u}_{k: k+r}+K_{k} \tilde{y}_{k: k+r}$

$\tilde{P}_{k+1}=\left(\bar{A}_{k}+\bar{I}_{n_{x}} X_{k} M_{k} \bar{B}_{k}\right) \tilde{P}_{k}\left(\bar{A}_{k}+\bar{I}_{n_{x}} X_{k} M_{k} \bar{B}_{k}\right)^{T}+\left(\bar{C}_{k}+\bar{I}_{n_{x}} X_{k} M_{k} \bar{D}_{k}\right) \Sigma_{k+r}\left(\bar{C}_{k}+\bar{I}_{n_{x}} X_{k} M_{k} \bar{D}_{k}\right)^{T}$

$\%$ UI Observer

$J_{k}=U_{r, n_{d}} \mathscr{D}_{k: k+r}^{\dagger}$

$P_{\epsilon_{k}}=\bar{E}_{k} \tilde{P}_{k} \bar{E}_{k}^{T}+\bar{F}_{k} \Sigma_{k+r} \bar{F}_{k}^{T}$

$\mathscr{S}_{k}=H_{k} P_{\epsilon_{k}} H_{k}^{T}$

Determine of $N_{k}$ according to remark 5.3

$Z_{k}=-J_{k} P_{\epsilon_{k}} H_{k}^{T} N_{k}^{T}\left(N_{k} \mathscr{S}_{k} N_{k}^{T}\right)^{-1}$

$L_{k}=J_{k}+Z_{k} N_{k} H_{k}$

$z_{k}=\tilde{y}_{k: k+r}-\mathscr{B}_{k: k+r}-\mathscr{A}_{k: k+r} \hat{x}_{k}$

$\hat{d}_{k}=L_{k} z_{k}$

If needed, calculate $P_{d_{k}}$ thanks to equation (5.6)

end for

\section{Practical implementation}

\subsection{Algorithm}

Algorithm 6.1 gives a practical method for implementing the state and UI observers described in Sections 4 and 5.

\subsection{Real time consideration}

It is worth noting that due to the use of $y_{k: k+r}$ in the observers, the estimation of $k+1$ can only be made after $k+r$ iterations. Hence, if $r \geqslant 2$, a delay of $(k+r)-(k+1)=r-1$ time steps is introduced in the estimation process (note that such a delay is also introduced in the filters provided in Sundaram \& Hadjicostis, 2006 and in Hsieh, 2009b). 
For some systems, such a delay can be very prejudicial, as controlling time-delayed systems is very difficult (ususally more difficult than controlling uncertain systems (Richard, 2003)). For such systems, the proposed observer cannot be used if $r \geqslant 2$.

Fortunately, not all applications are concerned by such an issue. Indeed, for many applications the estimation can be done with a slight delay without any consequence, or the estimation can even be done offline. In those cases, the proposed approach is totally satisfying.

Anyway, to the best of the authors' knowledge, when Assumption 4.1 is not satisfied for $r=1$, there does not exist any unbiased observer with minimum variance without a delay.

\subsection{Extension to linear parameter varying case}

Let consider the following linear parameter varying system:

$$
\left\{\begin{array}{l}
x_{k+1}=A_{\rho_{k}} x_{k}+B_{\rho_{k}} u_{k}+D_{\rho_{k}} d_{k}+F_{\rho_{k}} w_{k}, \\
y_{k}=C_{\rho_{k}} x_{k}+E_{\rho_{k}} d_{k}+v_{k}
\end{array},\right.
$$

whose the only difference with system (3.1) is the fact that all system matrices do not depend explicitly on time, but depend on a time-varying parameter $\rho_{k}$. In the sequel, the parameter $\rho_{k}$ is assumed to be known in real-time, and to belong to a compact set $\Omega$ of $\mathbb{R}^{n_{\rho}}$.

The results presented in the previous sections can be easily extended to such systems. In particular, if Algorithm 1 is used inline, it is just needed to provide it the successive values of the parameter. Besides, Assumptions 4.1 and 5.1 are replaced by Assumptions 6.1 and 6.2, respectively.

Assumption 6.1 There exist an integer $r \geqslant 1$ such that for any $\rho_{k: k+r} \in \Omega^{r+1}$ the following rank condition holds:

$$
\operatorname{rank}\left(\mathscr{D}_{\rho_{k: k+r}}\right)=\operatorname{rank}\left(\mathscr{D}_{\rho_{k: k+r}}\right)+\operatorname{rank}\left(\left[\begin{array}{c}
D_{\rho_{k}} \\
E_{\rho_{k}}
\end{array}\right]\right) .
$$

Assumption 6.2 It exists an integer $r \geqslant 1$ such that for any $\forall \rho_{k: k+r} \in \Omega^{r+1}$ the following rank condition is satisfied:

$$
\operatorname{rank}\left(\mathscr{D}_{\rho_{k: k+r}}\right)=\operatorname{rank}\left(\mathscr{D}_{\rho_{k: k+r}}\right)+n_{d} .
$$

The statements of Assumptions 6.1 and 6.2 are justified by the fact that the rank conditions have to be satisfied for any sequence of the parameter values.

\section{Illustrative examples}

In this section, three examples are proposed in order to illustrate the main results provided in the paper.

\subsection{Example 1}

Let consider the following system (from Sundaram \& Hadjicostis, 2006 and Hsieh, 2009a):

$$
\left\{\begin{array}{l}
x_{k+1}=\left[\begin{array}{cc}
0.1 & 1 \\
0 & 0.2
\end{array}\right] x_{k}+\left[\begin{array}{ll}
1 & 1 \\
0 & 1
\end{array}\right] d_{k}+w_{k} \\
y_{k}=\left[\begin{array}{ll}
0 & 1 \\
1 & 1
\end{array}\right] x_{k}+\left[\begin{array}{ll}
0 & 0 \\
0 & 1
\end{array}\right] d_{k}+v_{k}
\end{array},\right.
$$


TABLE 1 Example 1. RMSE (average on 1000 simulations) of the HO, SHO and MIVO

\begin{tabular}{lcccc}
\hline RMSE on $\ldots$ & $x_{1}$ & $x_{2}$ & $d_{1}$ & $d_{2}$ \\
\hline SHO & 0.2267 & 0.1991 & $N A$ & $N A$ \\
HO & 0.2273 & 0.2004 & 0.3095 & 0.2294 \\
MIVO & 0.2267 & 0.1991 & 0.3088 & 0.2267 \\
\hline
\end{tabular}

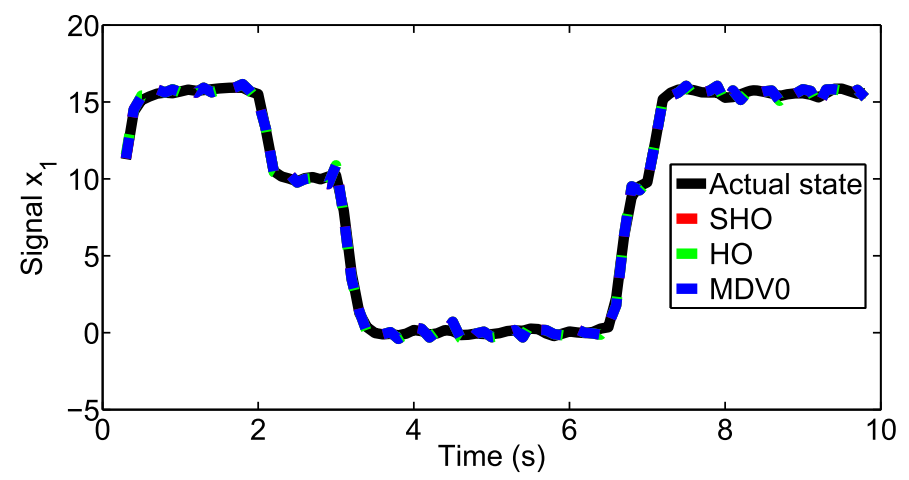

Estimation of the state $x_{1}$.

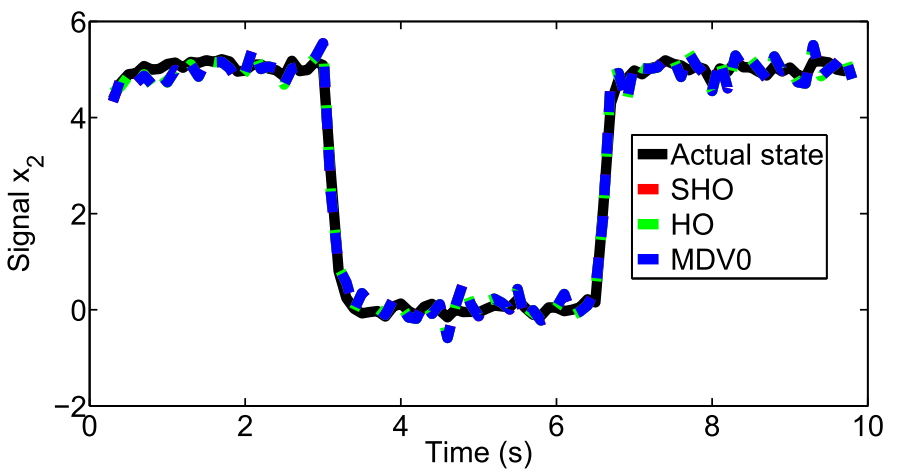

Estimation of the state $x_{2}$.

FIG. 1. Example 1. State estimation.

where $w_{k}$ and $v_{k}$ are Gaussian noises with zero mean and covariance matrices equal to $W=$ $\operatorname{diag}(0.01,0.01)$ and $V=\operatorname{diag}(0.04,0.04)$, respectively. As in Hsieh $(2009 \mathrm{~b})$, the UI is set to $d_{k}=$ $\left[\begin{array}{l}5 h[k-1]-5 h[k-20]+5 h[k-70] \\ 4 h[k-1]-4 h[k-30]+4 h[k-65]\end{array}\right]$, where $h$ is the Heaviside function. Here the rank condition is satisfied for $r=2$. The observers are used in order to estimate both state and UI. The results are presented on Figs 1 for the state estimation, and 2 for the UI estimation. Table 1 gives the root-mean-square performances. The observers are denoted by Sundaram and Hadjicostis Observer (SHO) for the observer proposed in Sundaram \& Hadjicostis (2006), Hsieh Observer (HO) for the one proposed in Hsieh (2009b) and Meyer Ichalal and Vigneron Observer (MIVO) for the one proposed in the present paper.

It can be seen in Table 1 that the proposed observer gives results slightly equivalent than those given by Hsieh (2009a), and similar results to the one proposed in Sundaram \& Hadjicostis (2006). 


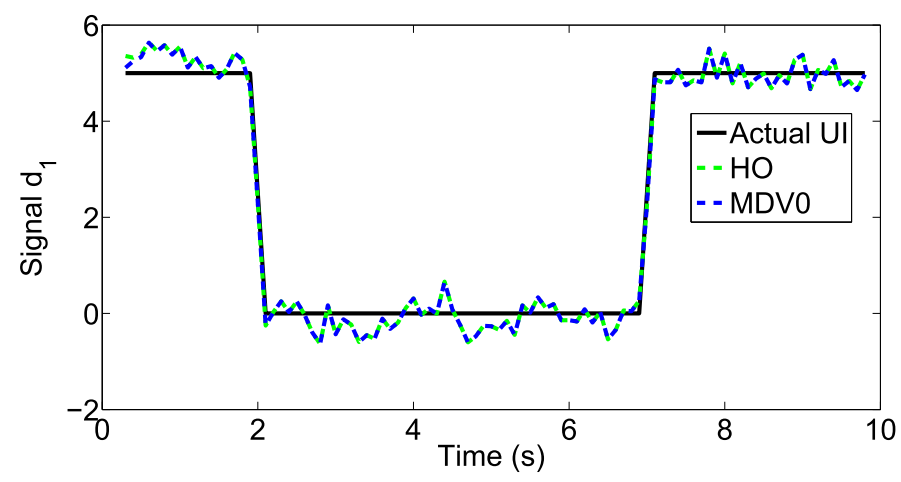

Estimation on Unknown Input $d_{1}$.

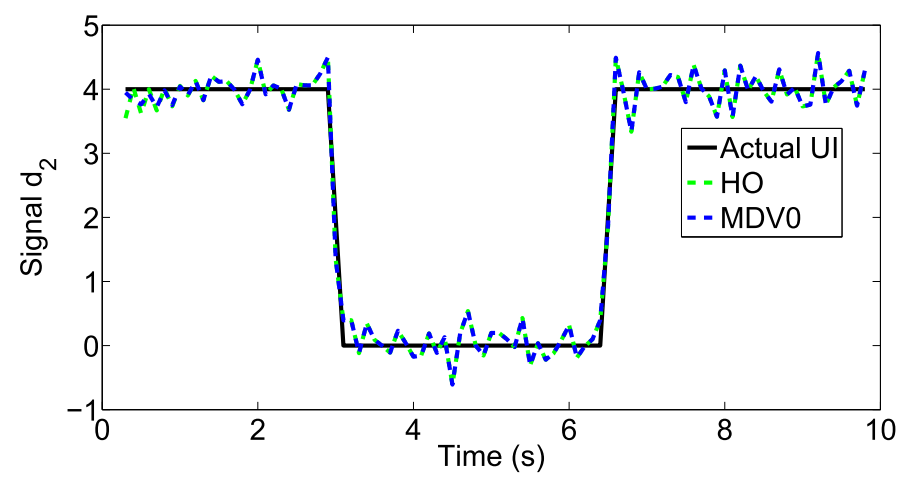

Estimation on Unknown Input $d_{2}$.

FIG. 2. Example 1. UI estimation.

As discussed in Remark 4.5, it can be concluded that, even if the three observers are constructed in different ways, they seem to give similar results. The few differences between the proposed observer and the one given by Hsieh (2009b) may be due to the difference of initialization that may affect the first estimations.

\subsection{Example 2}

In this second example, let us consider the following system (from Sundaram \& Hadjicostis, 2006 and Hsieh, 2009a):

$$
\left\{\begin{array}{l}
x_{k+1}=\left[\begin{array}{cccc}
0.1 & 0 & 0 & 0 \\
0 & 0.2 & 0 & 0 \\
0 & 0 & 0.3 & 0 \\
0 & 0 & 0 & 0.9
\end{array}\right] x_{k}+\left[\begin{array}{ll}
1 & 0 \\
0 & 1 \\
1 & 0 \\
0 & 1
\end{array}\right] d_{k}+w_{k} \\
y_{k}=\left[\begin{array}{cccc}
1 & 0 & 0 & 0 \\
-1 & 1 & 1 & -1
\end{array}\right] x_{k}+v_{k}
\end{array}\right.
$$


TABLE 2 Example 2. RMSE (average on 1000 simulations) of the HO, SHO and MIVO

\begin{tabular}{lcccccc}
\hline RMSE on $\ldots$ & $x_{1}$ & $x_{2}$ & $x_{3}$ & $x_{4}$ & $d_{1}$ & $d_{2}$ \\
SHO & 0.0998 & 0.2123 & 0.1020 & 0.1534 & $N A$ & $N A$ \\
HO & 0.0998 & 0.2123 & 0.1020 & 0.1535 & 0.1413 & 0.2585 \\
MIVO & 0.0998 & 0.2122 & 0.1020 & 0.1534 & 0.1413 & 0.2584 \\
\hline
\end{tabular}

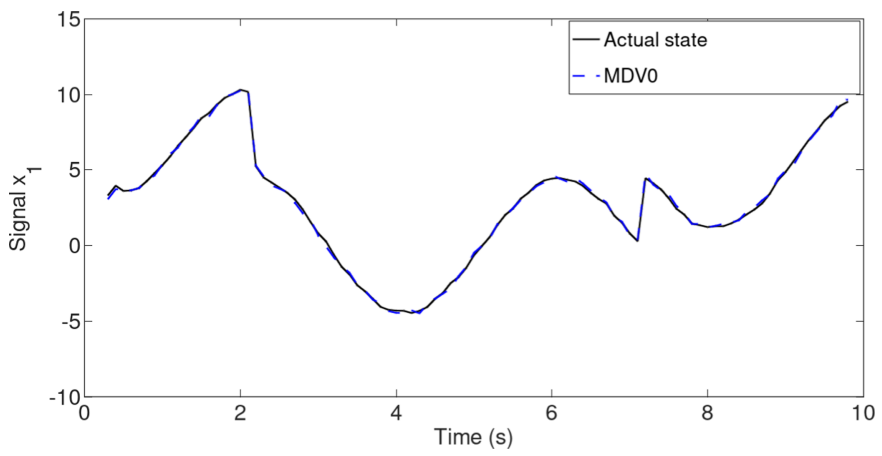

Estimation of the state $x_{1}$.

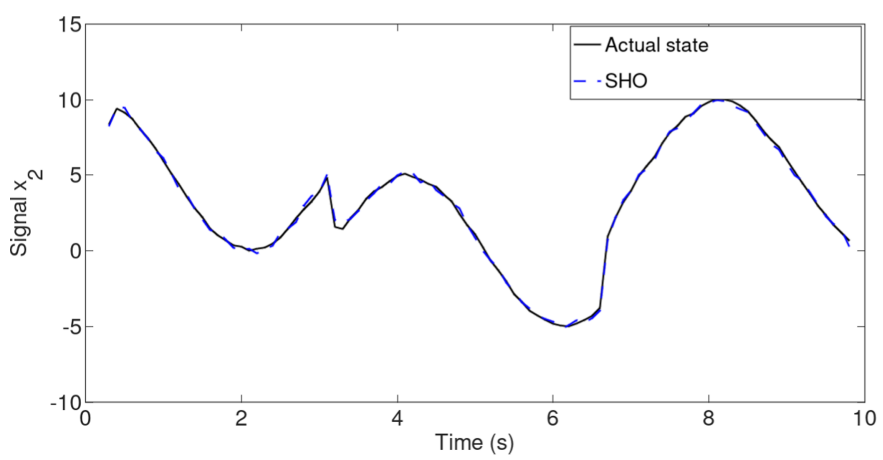

Estimation of the state $x_{2}$.

FIG. 3. Example 3. State estimation.

where $w_{k}$ and $v_{k}$ are Gaussian noises with zero mean and covariance matrices equal to $W=0.01 I_{4}$ and $V=0.01 I_{2}$, respectively. As in Hsieh (2009b), the UI is set to $d_{k}=\left[\begin{array}{c}\sin (0.01 k)+0.2 \sin (0.03 k) \\ 0.1 \sin (0.005 k)+0.1 \sin (0.05 k)\end{array}\right]$.

Here the rank condition is again satisfied for $r=2$. Note that for this example, Hsieh (2009b) uses another procedure than in the previous example in order to derive its estimations (as $E=0$ ), whereas in our case, the same observer is used, which has an advantage of unification for real applications.

Then the observers are used in order to estimate both state and UI. The results are presented in Table 2.

Here again, the three observers give quite similar results. 


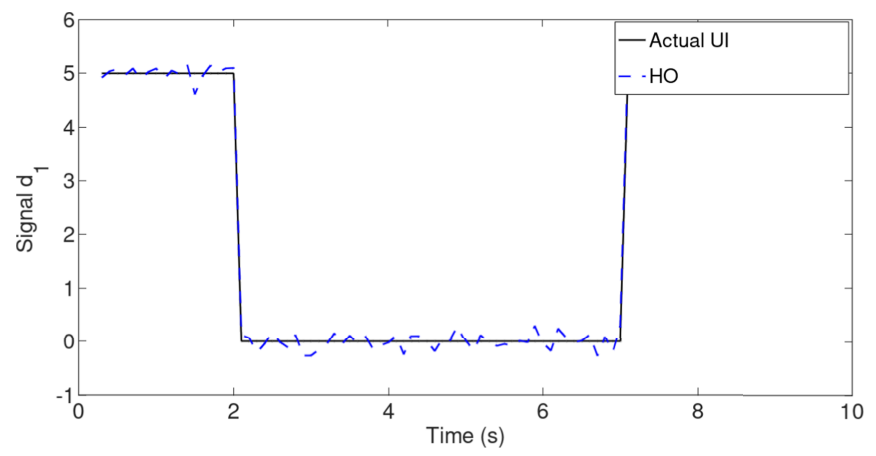

Estimation on Unknown Input $d_{1}$.

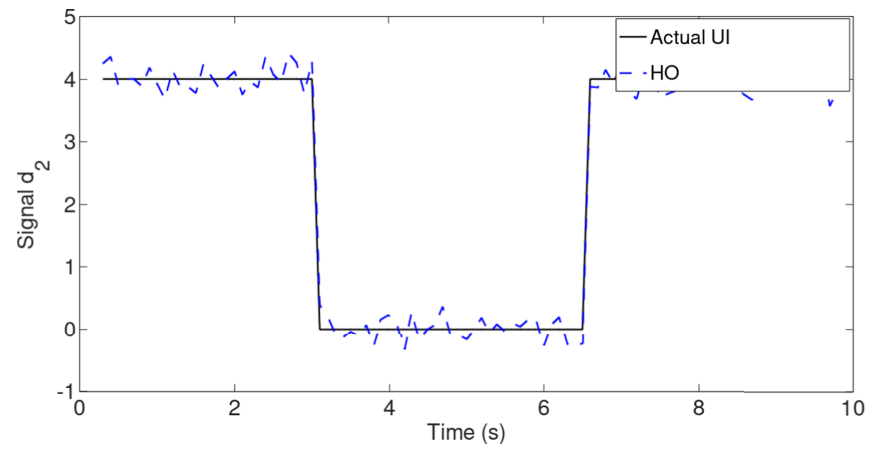

Estimation on Unknown Input $d_{2}$.

FIG. 4. Example 3. UI estimation.

\subsection{Example 3}

In this third example, let consider a modified version of Example 1 in order to illustrate the exhaustive form of an observer dealing with system such as (3.1). In particular, a known input is added, and the matrices are time-varying.

$$
\left\{\begin{array}{l}
x_{k+1}=\left[\begin{array}{cc}
0.1 & \frac{1}{k^{2}+1} \\
0 & 0.2
\end{array}\right] x_{k}+\left[\begin{array}{cc}
1 & \exp (-k) \\
0 & 1
\end{array}\right] u_{k}+\left[\begin{array}{cc}
1 & \frac{1}{k+1} \\
0 & 1
\end{array}\right] d_{k}+w_{k} \\
y_{k}=\left[\begin{array}{cc}
0 & 1 \\
1+\sin \left(k^{2}\right) & 1
\end{array}\right] x_{k}+\left[\begin{array}{ll}
0 & 0 \\
0 & 1
\end{array}\right] d_{k}+v_{k}
\end{array}\right.
$$

where $w_{k}$ and $v_{k}$ are Gaussian noises with zero mean and covariance matrices equal to $W=$ $\operatorname{diag}(0.01,0.01)$ and $V=\operatorname{diag}(0.04,0.04)$, respectively. The known input is set to $u_{k}=\left[\begin{array}{c}-4 \cos \left(\frac{\pi}{20} k\right) \\ 4 \cos \left(\frac{\pi}{20} k\right)\end{array}\right]$. Finally, as in Example 1, the UI is set to $d_{k}=\left[\begin{array}{l}5 h[k-1]-5 h[k-20]+5 h[k-70] \\ 4 h[k-1]-4 h[k-30]+4 h[k-65]\end{array}\right]$, where $h$ is the Heaviside function. 
TABLE 3 Example 3. RMSE (average on 1000 simulations) of the HO, SHO and MIVO

\begin{tabular}{lcccc}
\hline RMSE on $\ldots$ & $x_{1}$ & $x_{2}$ & $d_{1}$ & $d_{2}$ \\
\hline MIVO & 0.165 & 0.200 & 0.196 & 0.227 \\
\hline
\end{tabular}

Here the rank condition is satisfied for $r=2$. In this example, due to the presence of the known input, the observers provided in Sundaram \& Hadjicostis (2006) and Hsieh (2009b) cannot be used anymore (as this case has not been considered in those papers).

The observers provided in the present paper are used in order to estimate both state and UI. The results are presented on Figs 3 for the state estimation, and 4 for the UI estimation. Table 3 gives the root-mean-square performances.

It can be seen in Figs 3 and 4 that the state and UI estimations give quite good results (numerical values are given in Table 3), which confirm the theoretical contributions of the paper in a more complex example (with known input and time-varying matrices).

\section{Conclusions}

In that paper, the problem of state and input estimation is considered for an LTV system with UI and Gaussian noises in both state and measurement equations. The classical rank condition usually used to decouple the state estimation from the UI is relaxed in order to provide an observer that can be used in a wider range of systems. Observers are proved to be unbiased and with minimum variance error. Contrary to equivalent works on the subject, a unified UI observer is provided. Besides, a stability theorem is given for LTI systems, which is a novelty for minimum variance observer with relaxed rank condition. Examples are given in order to illustrate the results.

\section{REFERENCES}

Anderson, B. D. \& Moore, J. B. (1979) Optimal Filtering, vol. 21. Englewood Cliffs, pp. 22-95.

Ansari, A. \& Bernstein, D. S. (2018) Input estimation for nonminimum-phase systems with application to acceleration estimation for a maneuvering vehicle. IEEE Trans. Contr. Syst. Technol., 99, 1-12.

Charandabi, B. \& Marquez, H. (2014) A novel approach to unknown input filter design for discrete-time linear systems. Automat. J. IFAC, 50, 2835-2839.

Cheng, Y., Ye, H., Wang, Y. \& Zhou, D. (2009) Unbiased minimum-variance state estimation for linear systems with unknown input. Automat. J. IFAC, 45, 485-491.

Darouach, M. \& ZaSADZINSKI, M. (1997) Unbiased minimum variance estimation for systems with unknown exogenous inputs. Automat. J. IFAC, 33, 717-719.

Darouach, M., Zasadzinski, M. \& Boutayeb, M. (2003) Extension of minimum variance estimation for systems with unknown inputs. Automat. J. IFAC, 39, 867-876.

GilliJns, S. \& Moor, B. D. (2007a) Unbiased minimum-variance input and state estimation for linear discrete-time systems. Automat. J. IFAC, 43, 111-116.

GilliJNs, S. \& MoOR, B. D. (2007b) Unbiased minimum-variance input and state estimation for linear discrete-time systems with direct feedthrough. Automat. J. IFAC, 43, 934-937.

Grewal, M. S. (2011) Kalman filtering. New York, USA: Springer.

Hsien, C.-S. (2009a) Extension of unbiased minimum-variance input and state estimation for systems with unknown inputs. Automat. J. IFAC, 45, 2149-2153.

HsIEH, C.-S. (2009b) Optimal time-delayed joint input and state estimation for systems with unknown inputs. Decision and Control, 2009 held jointly with the 2009 28th Chinese Control Conference. CDC/CCC 2009. Proceedings of the 48th IEEE Conference on IEEE. Shanghai, China: IEEE, 4426-4431. 
HsieH, C.-S. (2013) State estimation for descriptor systems via the unknown input filtering method. Automat. J. IFAC, 49, 1281-1286.

ICHALAL, D. \& MammaR, S. (2015) On unknown input observers for lpv systems. IEEE Trans. Ind. Electron., 62 , 5870-5880.

IChalal, D., Marx, B., Ragot, J. \& Maquin, D. (2009) State and unknown input estimation for nonlinear systems described by Takagi-Sugeno models with unmeasurable premise variables. 17th Mediterranean Conference on Control and Automation, MED'09. Thessaloniki, Greece: IEEE.

JAZwinski, A. H. (2007) Stochastic Processes and Filtering Theory. Dunkirk, USA: Dover Publication, Inc.

Julier, S. J. \& Uhlmann, J. K. (1997) New extension of the kalman filter to nonlinear systems. Signal processing, sensor fusion, and target recognition VI. Orlando, USA: International Society for Optics and Photonics, pp. 182-194.

Kalman, R. (1960) A new approach to linear filtering and prediction problems. Trans. ASME J. Basic Eng., 82, $35-45$.

Li, W., Pan, J., Li, Y., Pan, S. \& LiU, Y. (2018) A kalman filtering for linear discrete-time system with unknown inputs. 2018 Chinese Control And Decision Conference (CCDC). IEEE, 5423-5428.

Luenberger, D. (1971) An introduction to observers. IEEE Trans. Autom. Control, 16, 596-602.

Maes, K., Gillijns, S. \& Lombaert, G. (2018) A smoothing algorithm for joint input-state estimation in structural dynamics. Mech. Syst. Signal Process., 98, 292-309.

Pertew, A. M., Marquez, H. J. \& Zhao, Q. (2005) $\mathcal{H}_{-} \infty$ synthesis of unknown input observers for nonlinear Lipschitz systems. Int. J. Control, 78, 1155-1165.

Petersen, K. B. \& Pedersen, M. S. (2008) The Matrix Cookbook, vol. 7. Technical University of Denmark, 15.

Richard, J.-P. (2003) Time-delay systems: an overview of some recent advances and open problems. Automat. J. IFAC, 39, 1667-1694.

Song, X. \& ZHENG, W. X. (2018a) Linear estimation for discrete-time periodic systems with unknown measurement input and missing measurements. ISA Trans.

Song, X. \& Zheng, W. X. (2018b) Some results on simultaneous input and state estimation for linear systems. IFAC-PapersOnLine, 51, 49-54.

Sundaram, S. \& Hadjicostis, C. N. (2005) Comments on 'time-delayed state estimator for linear systems with unknown inputs. Int. J. Control Automat. Syst., 3, 646-647.

Sundaram, S. \& Hadjicostis, C. N. (2006) Optimal state estimators for linear systems with unknown inputs. Proceedings of the 45th IEEE Conference on Decision and Control. IEEE, pp. 4763-4768.

Sundaram, S. \& Hadjicostis, C. N. (2007) Delayed observers for linear systems with unknown inputs. IEEE Trans. Automat. Control, 52, 334-339.

WANG, H., ZhaO, J., Xu, Z. \& Shao, Z. (2015) Input and state estimation for linear systems with a rank-deficient direct feedthrough matrix. ISA Trans., 57, 57-62.

YANG, J., Chen, Y. \& YU, K. (2014) State and unknown information estimation for non-linear systems with both input uncertainty and output disturbance. IMA J. Math. Control Inform., 33, 427-439.

Yong, S. Z., Zhu, M. \& Frazzoli, E. (2014) Simultaneous input and state smoothing for linear discrete-time stochastic systems with unknown inputs. Decision and Control (CDC), 2014 IEEE 53rd Annual Conference on IEEE. IEEE, 4204-4209.

Yong, S. Z., Zhu, M. \& Frazzoli, E. (2016) A unified filter for simultaneous input and state estimation of linear discrete-time stochastic systems. Automat. J. IFAC, 63, 321-329. 